\title{
Cubic optical nonlinearity of free electrons in bulk gold
}

\author{
N. I. Zheludev, P. J. Bennett, H. Loh, S. V. Popov, I. R. Shatwell, and Yu. P. Svirko* \\ Department of Physics, University of Southampton, Southampton SO17 1BJ, UK \\ V. E. Gusev ${ }^{\dagger}$ \\ Department Natuurkunde, Katholieke Universiteit Leuven, Celestijnenlaan 200D, B-3001, Leuven, Belgium \\ V. F. Kamalov ${ }^{\ddagger}$ and E. V. Slobodchikov \\ Institute of Chemical Physics, 4 Kosigina Street, Moscow 117977, Russia
}

Received March 6, 1995

\begin{abstract}
A fast $\left(\tau_{\text {response }}<90 \mathrm{fs}\right)$ free-electron spin-flipping frequency-degenerate nonlinearity with a significant value of $\left|\chi_{x x y y}^{(3)}(\omega, \omega, \omega,-\omega)-\chi_{x y y x}^{(3)}(\omega, \omega, \omega,-\omega)\right| \sim 10^{-8}$ esu has been observed in bulk gold at $1260 \mathrm{~nm}$ by use of a new pump-probe polarization-sensitive technique.
\end{abstract}

It is often anticipated that bulk metals will not yield any significant nonlinearity $\chi^{(3)}(\omega, \omega, \omega,-\omega)$ owing to free electrons. Free electrons, the major agent in infrared absorption in metals, were expected to have no Kerr-type dipole nonlinearity unless they are confined in microparticles, and free-electron third-order multipole nonlinearities were estimated to be very small. $^{1,2}$ Although we do not doubt the essential truth of these findings, we point out that these studies did not account for any spin-related processes. Here we report the results of direct measurements of the cubic nonlinearity in bulk gold that is responsible for the incoherent specular inverse Faraday effect (SIFE). We found that, in the near-infrared region (at $\lambda=1260 \mathrm{~nm}$ ), this nonlinearity is rather high $\left(\sim 10^{-8} \mathrm{esu}\right)$ and very fast, with relaxation times shorter than $90 \mathrm{fs}$. We attribute this to a spin-flipping nonlinearity of free-electron absorption that is mainly imaginary. To the best of our knowledge this nonlinearity has not yet been considered in the case of metals. We need to emphasize that this nonlinearity is intraband and consequently should be distinguished from all interband nonlinearities, for example, those responsible for the coherent third-harmonic generation process ${ }^{3}$ and the major incoherent visible-range cubic optical nonlinearity that is due to resonant optical transitions from the $d$ states to the $s-p$ conduction band in the vicinity of the Fermi level. ${ }^{4,5}$

The reported characterization of bulk metals became possible as a result of the development of a new pumpprobe polarization-sensitive technique based on the incoherent SIFE. ${ }^{6,7}$ This is a pump-probe nonlinearoptical phenomenon in which the medium is stimulated by a strong, circularly polarized pump wave, resulting in the alteration of the polarization of a probe wave reflected from the surface (see Fig. 1). The SIFE uniquely complements conventional techniques used for measurements of nonlinear-optical properties of opaque materials, such as transient pump-probe reflectivity, ${ }^{4,5}$ reflective harmonic generation, ${ }^{4,8}$ and polarization-sensitive reflective second-harmonic generation in magnetized media, ${ }^{9,10}$ because it provides information about combinations of the $\chi^{(3)}$ tensor that are not accessible by use of these traditional methods. If $\varepsilon_{1}+i \varepsilon_{2}=|\varepsilon| \exp (i \beta)=n^{2}$ is the complex dielectric coefficient of a strongly absorbing medium, the incoherent contribution to the pump-induced alteration of the probe wave polarization azimuth $\alpha_{r}$ owing to the SIFE is

$$
\begin{aligned}
& \delta \alpha_{r}=\frac{32 \pi I}{c|1+n|^{2}} \\
& \times \operatorname{Im}\left\{\frac{\left[\chi_{x x y y}^{(3)}(\omega, \omega, \omega,-\omega)-\chi_{x y y x}^{(3)}(\omega, \omega, \omega,-\omega)\right]}{n\left(1-n^{2}\right)}\right\} .
\end{aligned}
$$

Thus measurement of $\delta \alpha_{r}$ yields the value of $\left[\chi_{x x y y}^{(3)}(\omega, \omega, \omega,-\omega)-\chi_{x y y x}^{(3)}(\omega, \omega, \omega,-\omega)\right]$. Equation (1) has been obtained from a generalization of the approach of Ref. 7 in the case of strong absorption. It is valid within the scope of metallo-optics for $\left|\chi^{(3)}\right| I \ll c|\varepsilon|$, where $I$ is the pump intensity.

We studied mirrorlike 180-200-nm-thick gold layers deposited upon glass substrates in a vacuum of $3 \times 10^{-6}$ Torr by evaporation of a $99.99 \%$-pure gold sample. The thickness of our samples exceeded the depth of the optical skin layer $(12 \mathrm{~nm})$ by more than 1 order of magnitude. The reflectivity of the samples at $\lambda=1260 \mathrm{~nm}$ was $97.7 \pm 0.3 \%$. A polarization-sensitive technique was used as described elsewhere. ${ }^{11} \mathrm{~A}$ femtosecond Cr:forsterite laser ( $\lambda=1260 \mathrm{~nm}$; Ref. 12) pumped by a $\mathrm{Nd}^{3+}$ :YAG laser was used as the optical source. The probe and pump pulses had a typical intensity ratio of $1: 5$ and were focused to a spot $35 \mu \mathrm{m}$ in diameter. The temporal resolution of the measurements was $90 \mathrm{fs}$, which was determined by the duration of the laser pulses $\tau_{p}$. Reflected probe polarization azimuth rotation was observed on the scale of $10^{-6}-10^{-5} \mathrm{rad}$ in the pump intensity range $0.1-1 \mathrm{GW} \mathrm{cm}^{-2}$. The direction of the change in rotation is reversed for a pump wave of the opposite handedness. The nonlinear-optical response was instantaneous within the time resolution available, and no transient dynamics were observed [see 


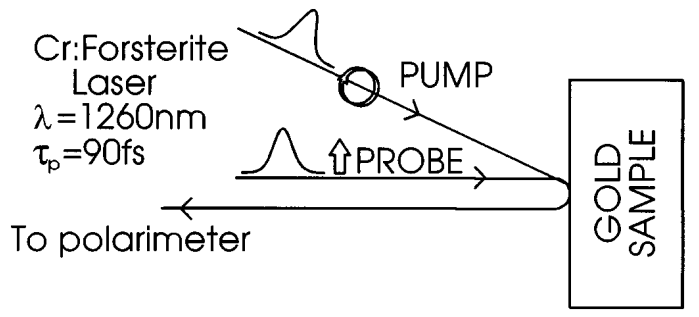

Fig. 1. Schematic of the arrangement to measure the SIFE in gold.

Fig. 2(a)]. A huge value for the material's dielectric constant in the infrared region [the Fresnel factor $|1+n|^{2} n\left(1-n^{2}\right) \sim|\varepsilon|^{2.5}=3.8 \times 10^{4}$ in Eq. (1) for gold at $1260 \mathrm{~nm}$ ] dramatically reduces the observable magnitudes of the reflective nonlinear phenomena, although the nonlinearity of the material is quite large. For the observed specific induced polarization azimuth rotation $\partial\left(\delta \alpha_{r}\right) / \partial I=1.1 \times 10^{-14} \mathrm{~W}^{-1} \mathrm{~cm}^{2}$ [see Fig. 2(b)], the corresponding nonlinearity is $\left[\chi_{x x y y}^{(3)}(\omega, \omega, \omega,-\omega)-\chi_{x y y x}^{(3)}(\omega, \omega, \omega,-\omega)\right] \simeq$ $1.5 \times 10^{-8}$ esu.

The incoherent SIFE may be treated by use of a conventional metallo-optics approach. The light reflected from a metallic surface gains a phase retardation $\alpha=\tan ^{-1}\left[2 \kappa /\left(n^{2}-1+\kappa^{2}\right)\right]$, where $2 n \kappa=\operatorname{Im}(\varepsilon)$ and $n^{2}-\kappa^{2}=\operatorname{Re}(\varepsilon)$. The polarization rotation of the reflected probe occurs as the result of the pump-induced circular differential retardation $\delta \alpha_{r}=\alpha_{+}-\alpha_{-}$. In the infrared region, where $|\kappa| \gg|n|$,

$$
\delta \alpha_{r} \approx-2 \frac{\left(\kappa_{+}-\kappa_{-}\right)}{\kappa^{2}}
$$

In the SIFE, circular dichroism is induced by a circularly polarized pump. Stimulation with an intensity of $500 \mathrm{MW} \mathrm{cm} \mathrm{cm}^{-2}$ yields a $\left(\kappa_{+}-\kappa_{-}\right) / \kappa$ of approximately $2 \times 10^{-5}$.

In the infrared (1260 nm, $\left.\hbar \omega_{L} \sim 0.98 \mathrm{eV}\right)$ the pump quantum energy is not sufficient to promote interband transitions. Consequently interband nonlinearities ${ }^{1-5}$ and, in particular, the Fermi-smearing nonlinearity owing to the resonant optical transitions from the $d$ states to the $s-p$ conduction band in the vicinity of the Fermi level, are not relevant to our case. All the nonlinearity shall be attributed to the free electrons. Multipole contributions to the free-electron nonlinearity ${ }^{13}$ are very small; from classical considerations that ignore spin-related phenomena we estimate them to be $10^{-14}$ esu, which cannot explain the observed SIFE. However, nonlinearity of optical orientation of the free electrons' spins is sufficient to explain the effect, and here we produce an estimate of this contribution.

From conservation of momentum and energy, freeelectron absorption occurs when an electron in the conductivity band absorbs a photon and simultaneously emits or absorbs a phonon or scatters on an impurity, defect, or boundary. To conserve angular momentum, the electron must change its spin orientation when a quantum from the circularly polarized pump wave is absorbed. In the SIFE, right- and left-handed circu- larly polarized light of the pump is absorbed by electrons with opposite spin-projection quantum numbers $m_{s}=1 / 2$ and $m_{s}=-1 / 2$. Here the quantization axis is in the direction of the light-wave vector. Thus the absorption of circularly polarized light effectively gives rise to an imbalance in the different spin occupancies $f_{+}\left(\varepsilon, m_{s}=1 / 2\right)$ and $f\left(\varepsilon, m_{s}=-1 / 2\right)$ at the electron energy level $\varepsilon$. In the infrared the optical quantum energy is much less than the Fermi energy of degenerate electrons, $\varepsilon_{F} \sim 5.5 \mathrm{eV}$, and the circular dichroism may be treated by use of the standard quantum approach to free-carrier absorption, ${ }^{14}$ which we have modified to account for the spin-dependent processes:

$$
\begin{aligned}
\frac{\kappa_{+}-\kappa_{-}}{\kappa} & =\frac{1}{\hbar \omega_{L}} \int \mathrm{d} \varepsilon\left\{\left[f_{-}(\varepsilon)-f_{+}(\varepsilon)\right]\right. \\
& -\left[f_{-}\left(\varepsilon+\hbar \omega_{L}\right)-f_{+}\left(\varepsilon+\hbar \omega_{L}\right)\right] \\
& \left.-2\left[f_{-}(\varepsilon) f_{+}\left(\varepsilon+\hbar \omega_{L}\right)-f_{+}(\varepsilon) f_{-}\left(\varepsilon+\hbar \omega_{L}\right)\right]\right\} .
\end{aligned}
$$

As a result of the pump absorption the initial stationary Fermi-Dirac distribution $f_{ \pm} \propto 1-\theta\left(\varepsilon-\varepsilon_{F}\right)$ is modified ( $\theta$ is a step function): the electron occupancies $f_{-}$homogeneously diminish below the Fermi level $\left(\varepsilon_{F}-\hbar \omega_{L} \leq \varepsilon \leq \varepsilon_{F}\right)$ while $f_{+}$increase above the Fermi level $\left(\varepsilon_{F} \leq \varepsilon \leq \varepsilon_{F}+\hbar \omega_{L}\right){ }^{14,15}$ In gold the electronphonon collisions do not significantly smooth this multistep distribution function within the time interval of interest ( $<90 \mathrm{fs}$ ); that is, the electron distribution is nonequilibrium. Indeed, following Ref. 16, even taking into account phonons with a maximum energy of $\hbar \omega_{D} \sim 14 \mathrm{meV}$ and a typical collision time of $\tau_{e-p} \sim 20 \mathrm{fs}$, the smoothing that is due to electron-phonon collisions during 90 fs spreads over only $70 \mathrm{meV}$, i.e., much less than the entire spectral width of the step $(980 \mathrm{meV})$. Also, with a characteristic electron-electron collision time of $\tau_{0} \sim 3 \mathrm{fs},{ }^{17}$ electronelectron collisions in the spectral region of interest $\left(\left|\varepsilon-\varepsilon_{F}\right|<\hbar \omega_{L}\right)$ are quite rare, with a characteristic time $\tau_{e-e} \sim \tau_{0}\left(\varepsilon_{F} /\left|\varepsilon-\varepsilon_{F}\right|\right)^{2} \sim 90$ fs. We neglected this process in this first-order estimate. Since the pump-induced change in occupancy of the electrons is

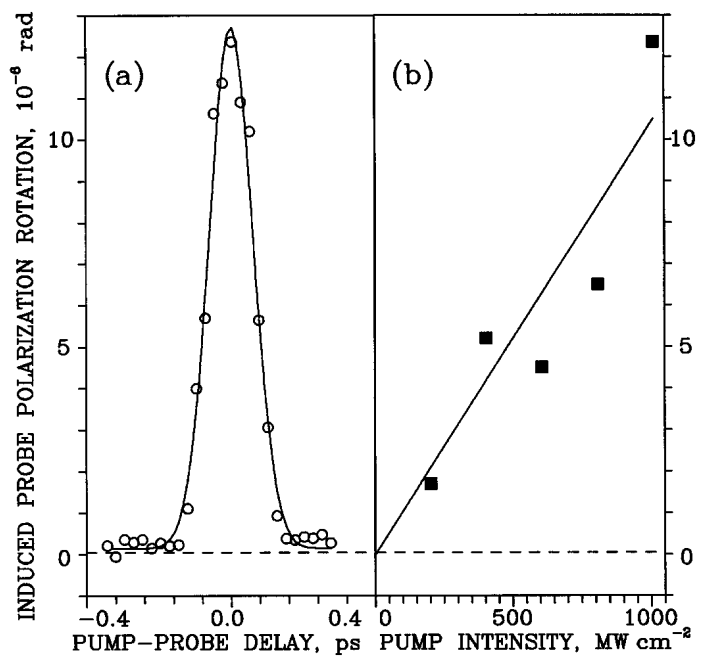

Fig. 2. SIFE rotation in gold ( $\lambda=1260 \mathrm{~nm}$, room temperature) versus (a) pump-probe delay $\left(I \sim 1 \mathrm{GW} \mathrm{cm}^{-2}\right)$ and (b) pump pulse intensity (zero pump-probe delay). 
small ( $\sim 10^{-4}-10^{-3}$ in our experiments), Eq. (3) may be linearized:

$$
\frac{\kappa_{+}-\kappa_{-}}{\kappa}=2 \frac{N_{-}-N_{+}}{\rho\left(\varepsilon_{F}\right) \hbar \omega_{L}} .
$$

Here $\rho\left(\varepsilon_{F}\right)$ is the density of states; $N_{ \pm}$are the concentrations of electrons with the energies $\varepsilon$, where $\left|\varepsilon-\varepsilon_{F}\right| \leq \hbar \omega_{L}$ and spin projections $m_{s}= \pm 1 / 2$ correspondingly. For $\delta N=N_{-}-N_{+}$, at the irradiated surface we have derived the following balance equation:

$$
\frac{\delta}{\delta t}(\delta N)=-\frac{2}{\tau_{s}}(\delta N)+\frac{\xi(1-R)}{\hbar \omega_{L}}\left(2-\frac{\tau}{\tau_{S}}\right) I,
$$

which accounts for fractional absorption of the pump with intensity $I$ and for spin relaxation. Here $\xi=L^{-1}=2 \omega \kappa / c$ is the light absorption coefficient, $R$ is reflectivity, and $\tau_{s}$ is a characteristic spin relaxation time that is due to electron collisions with photons, dislocations, etc. In Eq. (5), $\tau$ is the relaxation time for electron momentum transfer that accounts for both spin-flipping $\left(\tau_{s}\right)$ and spin-preserving $\left(\tau_{n}\right)$ scattering: $\tau^{-1}=\tau_{s}^{-1}+\tau_{n}^{-1}$. Equation (5) neglects the influence of diffusion on the dynamics of $\delta N$. This is a justifiable assumption since the characteristic time of electron diffusion $\tau_{D}$ through the skin layer of thickness $L \sim 12 \mathrm{~nm}$ significantly exceeds $\tau_{s}$. Indeed, with the Fermi velocity of electrons in gold $V_{F} \simeq 1.4 \times 10^{8} \mathrm{~cm} \mathrm{~s}^{-1}$ and the electron diffusivity $D \sim(1 / 3) V_{F}^{2} \tau$, the electron diffusion time is $\tau_{D} \sim L^{2} / D \sim 95 \mathrm{fs}$. To derive the final formulas we use the relationship between the reflectivity of light and the plasmon frequency, $1-R=2 /\left(\tau \omega_{p}\right)$, where $\omega_{p}=\left(4 \pi e^{2} N / m^{*}\right)^{1 / 2}$, and $m^{*}$ and $N$ denote the effective free-electron mass and concentration:

$$
\delta N \simeq \frac{\alpha(1-R) \tau_{S}}{\hbar \omega_{L}}\left(1-\frac{\tau}{2 \tau_{S}}\right) I .
$$

From Eqs. (2) and (4)-(6), taking into account the inhomogeneity of excitation, we obtain

$$
\left|\frac{\delta \alpha_{r}}{\delta I}\right|_{\text {theor }} \simeq \frac{8}{c \hbar \omega_{p} \rho\left(\varepsilon_{F}\right) \hbar \omega_{L}}\left(\frac{\tau_{S}}{\tau}-\frac{1}{2}\right) .
$$

With $\omega_{p} \sim 1.4 \times 10^{16} \mathrm{~s}^{-1}, N \simeq 5.9 \times 10^{22} \mathrm{~cm}^{-3}$, and $m^{*}$ being close to the free-electron mass $m_{0}$, we get $\hbar \omega_{p} \simeq 9 \mathrm{eV}$ and $\rho\left(\varepsilon_{F}\right)=3 N /\left(4 \varepsilon_{F}\right) \simeq 8 \times 10^{21} \mathrm{eV}^{-1} \mathrm{~cm}^{-3}$. Relation (7) agrees with the experimentally observed magnitude of the rotation for $\left(\tau_{S} / \tau\right)_{\exp } \simeq 1$; i.e., nearly every electron momentum relaxation collision causes spin flipping. The value of $\tau_{S} / \tau$ found is in good agreement with theoretical estimates for the acoustic-type large-angle electron scattering by elastic strain fields in gold: $\tau_{S} / \tau \approx(1 / 2)\left(\Delta g \varepsilon_{0} / d\right)^{-2} \approx 3.6 .{ }^{17}$ Here $d$ is the electron-phonon deformation potential $d=(2 / 3) \varepsilon_{F}, \varepsilon_{0}$ is the characteristic atomic energy $\varepsilon_{0}=m_{0} e^{4} / 2 \hbar^{2}$, and $\Delta g \approx 0.1$ is the shift of the electron $g$ factor in gold. We attribute some discrepancy between the experimen- tal value of $\tau_{S} / \tau$ and the above estimate to other nonacoustic-type scattering mechanisms; taking these into account should scale down the theoretically estimated values of $\tau_{S} / \tau$. We have not resolved the relaxation time for this spin-flipping nonlinearity, but the above analysis predicts it to be equal the spin relaxation time, i.e., to be on the scale of $10^{-14} \mathrm{~s}$.

In summary, we have observed significant incoherent infrared optical nonlinearities in bulk gold that we attribute to saturation of a light-stimulated spinflipping process.

This study was partially supported by Lasers for Photochemistry, Ltd., Moscow, the R. W. Paul Instrument Fund, the Royal Society (grant RSRG13703), the Engineering and Physical Sciences Research Council (grants GR/J26854 and GR/K14230), and the International Science Foundation (grant N4V000).

*Permanent address, General Physics Institute, 38 Vavilov Street, Moscow 117942, Russia.

$\dagger$ Permanent address, International Laser Center, Moscow State University, Moscow 119899, Russia.

$\mp$ Current address, Department of Chemistry and Biochemistry, Georgia Institute of Technology, Atlanta, Georgia 30332.

\section{References}

1. F. Hache, D. Ricard, and C. Flytzanis, J. Opt. Soc. Am. B 3, 1647 (1986).

2. F. Hache, D. Ricard, C. Flytzanis, and U. Kreibig, Appl. Phys. A 47, 347 (1988).

3. W. K. Burns and N. Bloembergen, Phys. Rev. B 4, 3437 (1971).

4. R. W. Schoenlein, W. Z. Lin, J. G. Fujimoto, and G. L. Eesley, Phys. Rev. Lett. 58, 1680 (1987).

5. G. L. Eesley, Phys. Rev. B 33, 2144 (1986).

6. S. V. Popov, N. I. Zheludev, and Yu. P. Svirko, Opt. Lett. 19, 13 (1994).

7. Yu. P. Svirko and N. I. Zheludev, J. Opt. Soc. Am. B 11, 1388 (1994).

8. See numerous references in Y. R. Shen, Surf. Sci. 300, 551 (1994); A. Liebsch, Surf. Sci. 309, 1007 (1994).

9. R. P. Pan, H. D. Wei, and Y. R. Shen, Phys. Rev. B 39, 1229 (1989).

10. W. Hübner and K. H. Bennemann, Phys. Rev. B 40, 5973 (1989).

11. N. I. Zheludev, S. V. Popov, A. Malinowski, Yu. Svirko, W. Y. Liang, and C. T. Lin, Solid State Commun. 90, 287 (1994).

12. A. A. Ivanov, V. F. Kamalov, A. P. Lifanov, J. Lucassen, B. I. Minkov, and E. V. Slobochikov, Kavantovaya Elektron. 20, 1039 (1993).

13. C. S. Wang, J. M. Chen, and J. R. Bower, Opt. Commun. 8, 275 (1973).

14. W. P. Dumke, Phys. Rev. 124, 1813 (1961).

15. W. S. Fann, R. Storz, H. W. K. Tom, and J. Boker, Phys. Rev. B 46, 13592 (1992).

16. F. Beuneu and P. Monod, Phys. Rev. B 18, 2422 (1978).

17. V. F. Gantmaher and I. B. Levinson, Scattering of Carriers in Metals and Semiconductors (Nauka, Moscow, 1984), Chap. 13. 